\title{
Spherical-multipole analysis of an arbitrarily directed complex-source beam diffracted by an acoustically soft or hard circular cone
}

\author{
A. Reinhardt ${ }^{1}$, H. Bruens ${ }^{1}$, L. Klinkenbusch ${ }^{1}$, M. Katsav ${ }^{2}$, and E. Heyman ${ }^{2}$ \\ ${ }^{1}$ Institute of Electrical and Information Engineering, Kiel University, Kaiserstr. 2, 24143 Kiel, Germany \\ ${ }^{2}$ School of Electrical Engineering, Tel Aviv University, Tel Aviv 69978, Israel
}

Correspondence to: L. Klinkenbusch (lbk@tf.uni-kiel.de)

Received: 8 January 2015 - Revised: 23 March 2015 - Accepted: 24 March 2015 - Published: 3 November 2015

\begin{abstract}
An analytical approach to analyze the diffraction of an arbitrarily directed complex-source beam (CSB) by an acoustically soft or hard semi-infinite circular cone is presented. The beam is generated by assigning a complexvalued location to a point source; its waist and direction are defined by the real and imaginary parts of the source coordinate, respectively. The corresponding scalar boundary-value problem is solved by a spherical-multipole analysis. The solution requires the calculation of associated Legendre functions of the first kind for complex-valued arguments which turns out to be a non-trivial task. Beside a numerical analysis of the corresponding algorithms we present numerical results for the total near- and scattered far-fields.
\end{abstract}

\section{Introduction}

A certain class of analytical solutions of the Helmholtz equation provides the basis of developing and improving asymptotic and hybrid methods such as the Geometrical Theory of Diffraction (GTD) or the Uniform Theory of Diffraction (UTD). Particularly, the scattering of electromagnetic waves by certain geometrical details such as tips, edges, corners, and curved surfaces are of importance. In that context the acoustic and electromagnetic scattering and diffraction of a plane wave by a semi-infinite circular cone has been investigated frequently (Bowman et al., 1987). By using a sphericalmultipole eigenfunction expansion of the cone for the total field and a free-space type multipole expansion of the scattered field, in Klinkenbusch (2007), Kijowski and Klinkenbusch (2011) solutions were derived which, however, suffered from a missing convergence of the finally obtained multipole series. Rather than using an incident full plane wave the new approach in Katsav et al. (2012), Brüns and Klinkenbusch (2013) started from a complex-source beam (CSB) as the incident field. One main advantage of this method is the ability to probe just the section of interest, for instance the area near to the cone's tip. Furthermore, the convergence problems mentioned above are avoided using a CSB. While Katsav et al. (2012), Brüns and Klinkenbusch (2013) investigated the scattering and diffraction of a CSB pointed directly towards the tip of the circular cone the present contribution deals with the more general case of an arbitrarily directed CSB illuminating any desired part of the cone.

\section{Formulation of the problem and spherical-multipole expansion}

Consider an acoustically soft (index "s" in the following) or hard (index "h") circular semi-infinite cone located symmetrically around the negative $z$ axis as depicted in Fig. 1. The tip is located in the origin, while the surface is described by the half outer opening angle $\vartheta_{0}$. At a time-factor $e^{+j \omega t}$, for a unit point source located at $\boldsymbol{r}_{\mathrm{c}}$ the phasor of the normalized time-harmonic scalar field $\Phi(\boldsymbol{r})$ has to satisfy the scalar Helmholtz equation

$\Delta \Phi(\boldsymbol{r})+\kappa^{2} \Phi(\boldsymbol{r})=-\delta\left(\boldsymbol{r}-\boldsymbol{r}_{\mathrm{c}}\right)$.

Here $\kappa=2 \pi / \lambda$ represents the wavenumber at a wave length $\lambda$. In case of an acoustically soft or hard cone the field has to fulfill the Dirichlet condition

$\left.\Phi_{\mathrm{S}}(\boldsymbol{r})\right|_{\vartheta=\vartheta_{0}}=0$ 


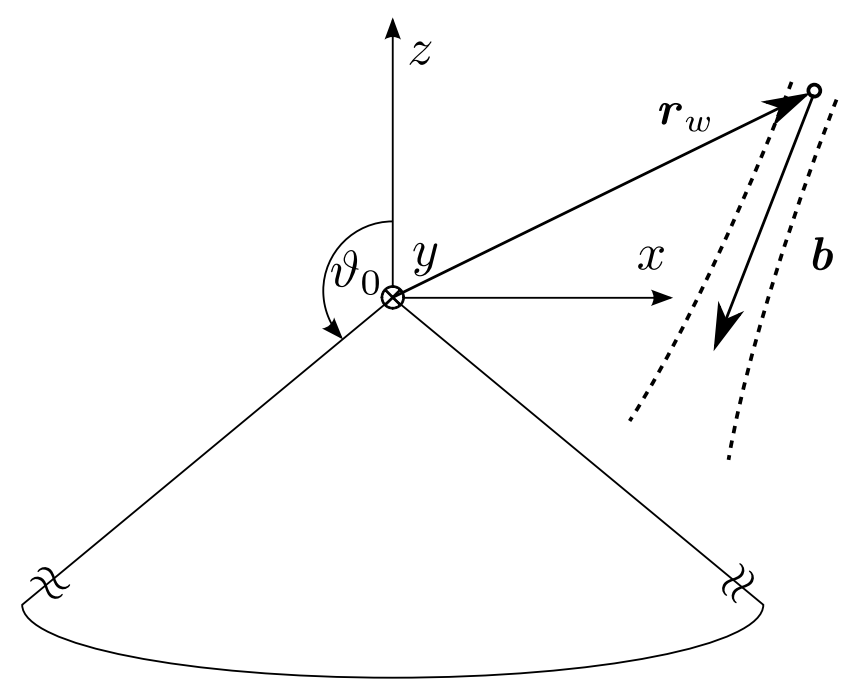

Figure 1. Semi-infinite circular cone illuminated by an arbitrarily oriented complex-source beam.

or the Neumann condition

$\left.\frac{\partial \Phi_{\mathrm{h}}(\boldsymbol{r})}{\partial \vartheta}\right|_{\vartheta=\vartheta_{0}}=0$

respectively. In spherical coordinates $r, \vartheta, \varphi$ with the $z$ axis as the polar axis, the standard (Bernoulli) separation ansatz with separation constants $\sigma_{\tau}\left(\sigma_{\tau}+1\right)$ and $m^{2}$ leads to

$\Phi_{\tau}(r, \vartheta, \varphi)=z_{\sigma_{\tau}}(\kappa r) Y_{\sigma_{\tau}}^{m}(\vartheta, \varphi)$

where $\tau \in\{s, h\}$ and $z_{\sigma_{\tau}}(\kappa r)$ and $Y_{\sigma_{\tau}}^{m}(\vartheta, \varphi)$ are spherical Bessel functions and normalized surface spherical harmonics, respectively. The spherical Bessel functions of order $\sigma_{\tau}$ are related to the ordinary Bessel functions $Z_{\sigma_{\tau}+\frac{1}{2}}(\kappa r)$ of or$\operatorname{der} \sigma_{\tau}+\frac{1}{2}$ by

$z_{\sigma_{\tau}}(\kappa r)=\sqrt{\frac{\pi}{2 \kappa r}} Z_{\sigma_{\tau}+\frac{1}{2}}(\kappa r)$

while the surface spherical harmonics of order $m$ and degree $\sigma_{\tau}$ are represented by

$Y_{\sigma_{\tau}}^{m}(\vartheta, \varphi)=N_{\sigma_{\tau}}^{m} P_{\sigma_{\tau}}^{m}(\cos \vartheta) e^{j m \varphi}$.

Here $P_{\sigma_{\tau}}^{m}(\cos \vartheta)$ is an associated Legendre function of the first kind while $N_{\sigma_{\tau}}^{m}$ is a normalization constant chosen such that

$\int_{0}^{\vartheta_{0}} \int_{0}^{2 \pi}\left|Y_{\sigma_{\tau}}^{m}(\vartheta, \varphi)\right|^{2} \sin \vartheta \mathrm{d} \vartheta \mathrm{d} \varphi=1$.

Finally, the normalized total field consists of a linear superposition of all elemental solutions of the scalar Helmholtz Eq. (1) which satisfy the boundary conditions. It can be represented by means of the spherical-multipole expansion (Bowman et al., 1987)

$$
\begin{aligned}
& \Phi_{\tau}\left(\boldsymbol{r}, \boldsymbol{r}_{\mathrm{c}}\right)= \\
& -j \kappa \sum_{\sigma_{\tau}, m} j_{\sigma_{\tau}}(\kappa r) h_{\sigma_{\tau}}^{(2)}\left(\kappa r_{\mathrm{c}}\right) Y_{\sigma_{\tau}}^{m}(\vartheta, \varphi) Y_{\sigma_{\tau}}^{m-}\left(\vartheta_{\mathrm{c}}, \varphi_{\mathrm{c}}\right) \\
& \quad \text { if }|r| \leq\left|r_{\mathrm{c}}\right| \\
& -j \kappa \sum_{\sigma_{\tau}, m} j_{\sigma_{\tau}}\left(\kappa r_{\mathrm{c}}\right) h_{\sigma_{\tau}}^{(2)}(\kappa r) Y_{\sigma_{\tau}}^{m}\left(\vartheta_{\mathrm{c}}, \varphi_{\mathrm{c}}\right) Y_{\sigma_{\tau}}^{m-}(\vartheta, \varphi) \\
& \quad \text { if }|r|>\left|r_{\mathrm{c}}\right|
\end{aligned}
$$

where $Y_{\sigma_{\tau}}^{m-}$ is defined as

$Y_{\sigma_{\tau}}^{m-}(\vartheta, \varphi)=N_{\sigma_{\tau}}^{m} P_{\sigma_{\tau}}^{m}(\cos \vartheta) e^{-j m \varphi}$.

Note that the common definition in the literature is $Y_{\sigma_{\tau}}^{m-}=$ $Y_{\sigma_{\tau}}^{m *}\left({ }^{*}\right.$ denotes the complex conjugation) which, however, is generally valid only in case of a real-valued argument $\vartheta$. Since the solution has to be regular everywhere and to satisfy the radiation condition, spherical Bessel functions of the first kind $j_{\sigma_{\tau}}(\kappa r)$ and spherical Hankel functions of the second kind $h_{\sigma_{\tau}}^{(2)}(\kappa r)$ are employed. The boundary conditions (2), (3) can be directly transferred to the associated Legendre functions of the first kind

$$
\begin{aligned}
\left.P_{\sigma_{\mathrm{s}}}^{m}(\cos \vartheta)\right|_{\vartheta=\vartheta_{0}} & =0, \\
\left.\frac{\partial P_{\sigma_{\mathrm{h}}}^{m}(\cos \vartheta)}{\partial \vartheta}\right|_{\vartheta}=\vartheta_{0} & =0,
\end{aligned}
$$

respectively. The eigenvalues $\sigma_{\mathrm{s}}, \sigma_{\mathrm{h}}$ can be determined by searching the zeros of Eqs. (10), (11) with a suitable numerical procedure such as the bisection method. Because the solutions are $2 \pi$ periodic in $\varphi$, the $m$ have to be integers $(m=0, \pm 1, \pm 2, \ldots)$. Moreover, as shown in Katsav et al. (2012) it holds that $m^{2} \leq \sigma_{\tau}\left(\sigma_{\tau}+1\right)$. Consequently there are discrete pairs of eigenvalues for $\left(\sigma_{\mathrm{s}}, m\right)$ for the soft cone and $\left(\sigma_{\mathrm{h}}, m\right)$ for the hard cone, respectively, as exemplarily represented in Fig. 2.

In the following we shall evaluate the spherical-multipole expansion of the cone (8) for a unit point source located at a complex location. That will yield the field for the cone illuminated by a complex-source beam (CSB) as outlined in Sect. 3. To give that CSB an arbitrary direction and thus to probe any desired area of the cone requires the evaluation of associated Legendre functions of the first kind of realvalued orders but for complex arguments which are discussed in Sect. 4. Corresponding numerical results are finally presented in Sect. 5.

\section{Complex-source beam}

The CSB offers the possibilty to describe a focussed beam analytically. If a complex valued location $\boldsymbol{r}_{\mathrm{c}}=\boldsymbol{r}_{\mathrm{w}}-j \boldsymbol{b}$ is assigned to a point source a beam is generated whose reference 


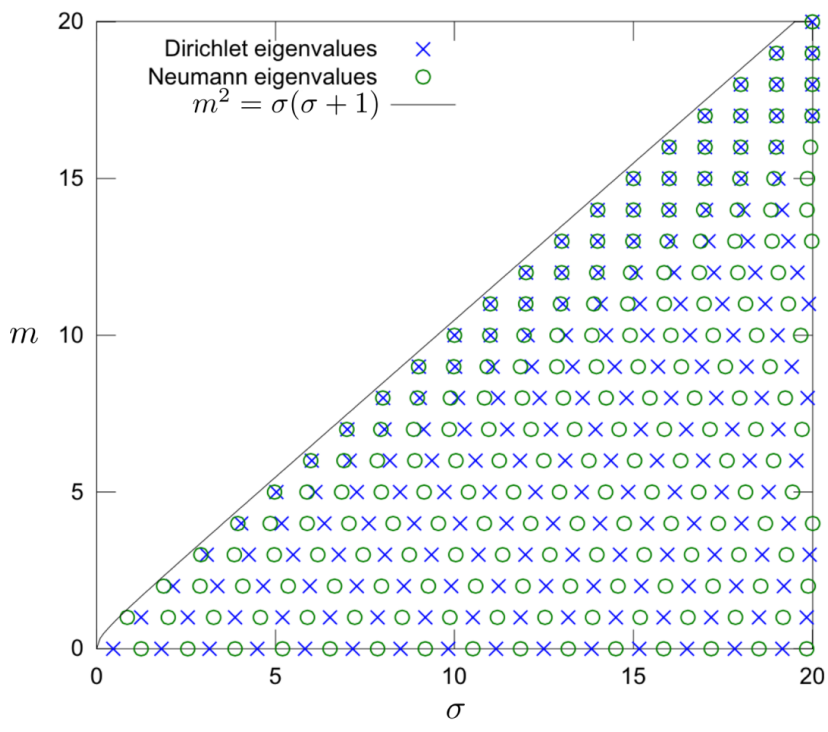

Figure 2. Eigenvalue pairs (soft/Dirichlet and hard/Neumann) for a cone with $\vartheta_{0}=140^{\circ}$.

point of the waist is defined by the real part $\boldsymbol{r}_{\mathrm{w}}$ and whose direction is defined by the imaginary part of the source coordinate $\boldsymbol{b}$ as shown in Fig. 1. It can be shown, that in a paraxial approximation the CSB describes a Gaussian beam (Felsen, 1976; Orlob and Peschel, 2010). For a beam directed towards the origin, i. e. the cone's tip, it has been shown that in spherical components only their radial part $r_{\mathrm{c}}$ is complex valued while the angular parts $\vartheta_{\mathrm{c}}, \varphi_{\mathrm{c}}$ remain real valued (Katsav et al., 2012). However, for an arbitrarily directed beam we have complex values for $\vartheta_{\mathrm{c}}, \varphi_{\mathrm{c}}$ in the multipole expansion (8). This requires the evaluation of the associated Legendre functions of the first kind for a complex-valued argument $\cos \vartheta_{\mathrm{c}}$.

\section{Computation of associated Legendre functions of the first kind for a complex-valued argument}

The associated Legendre functions of the first kind $P_{\sigma}^{m}(z)$ are the regular solutions of the differential equation

$\left(1-z^{2}\right) \frac{\mathrm{d}^{2} w}{\mathrm{~d} z^{2}}-2 z \frac{\mathrm{d} w}{\mathrm{~d} z}+\left(\sigma(\sigma+1)-\frac{m^{2}}{1-z^{2}}\right) w=0$.

Evaluating them in an efficient way may become a non-trivial task in practice since the structure of the series that defines the function creates many numerical issues such as cancellation and round-off errors especially for certain ranges of the parameters $\sigma, m$ and $z$. The most common computation algorithms feature the evaluation only for integer orders $m$, integer degrees $\sigma=n$ and real-valued arguments. For solving the boundary-value problem at hand (real-valued order and complex-valued argument) we present three alternative approaches.

The most obvious idea might be to apply a numerical standard method for solving differential equations. The best results taking into account accuracy and computation had been achieved by using a fourth order Runge-Kutta method (RK4).

As a second approach we applied the following integral representation derived from the Schlaefli integral

$P_{\sigma}(z)=\frac{1}{2 \pi} \int_{C} \frac{\left(1-s^{2}\right)^{\sigma}}{2^{\sigma}(s-z)^{\sigma+1}} \mathrm{~d} s$.

Note that the closed contour $C$ encircles the points $s=z$ and $s=1$ counterclockwise. More details are found in (Whittaker and Watson, 2004) page 307. Exploiting that for integer orders $m$ the associated Legendre functions of the first kind can be derived from (ordinary) Legendre functions by

$P_{\sigma}^{m}(z)=(-1)^{m}\left(1-z^{2}\right)^{m / 2} \frac{\mathrm{d}^{m}}{\mathrm{~d} z^{m}} P_{\sigma}(z)$

and with the substitution $s=z+\sqrt{1-z^{2}} e^{j \phi}(0 \leq \phi \leq 2 \pi)$, Eq. (13) can be transformed to

$$
\begin{gathered}
P_{\sigma}^{m}(z)=(-1)^{m} \frac{(\sigma+1)(\sigma+2) \ldots(\sigma+m)}{2 \pi} \\
\int_{0}^{2 \pi}\left(z+j \sin \phi \sqrt{1-z^{2}}\right)^{\sigma} e^{-j m \phi} \mathrm{d} \phi .
\end{gathered}
$$

This definite integral has been solved by a standard quadrature method.

The third approach was performed utilizing Ferrer's function

$$
\begin{aligned}
& P_{\sigma}^{m}(z)=(-1)^{m} \frac{\Gamma(\sigma+m+1)}{2^{m} \Gamma(\sigma-m+1)}\left(1-z^{2}\right)^{m / 2} \frac{1}{\Gamma(m+1)} \\
& { }_{2} F_{1}\left(\sigma+m+1, m-\sigma ; m+1 ; \frac{1}{2}-\frac{1}{2} z\right),
\end{aligned}
$$

that is, a representation of the associated Legendre function of the first kind by means of the Gaussian hypergeometric function

${ }_{2} F_{1}\left(a, b ; c ; z^{\prime}\right)=\sum_{k=0}^{\infty} \frac{(a)_{k}(b)_{k}}{(c)_{k}} \frac{z^{\prime k}}{k !}$.

Here, $(a)_{k}$ is representing the Pochhammer symbol defined for $k=0,1,2, \ldots$ by

$(a)_{k}=\frac{\Gamma(a+k)}{\Gamma(a)}$

with $\Gamma$ being the Gamma function.

While the problem gets shifted towards computing ${ }_{2} F_{1}\left(a, b ; c ; z^{\prime}\right)$, an algorithm was found based on the recurrence relation of the Gamma function $\Gamma(x+1)=x \Gamma(x)$ which leads to

$$
F_{k+1}=\frac{(a+k)(b+k)}{c+k} \frac{z^{\prime}}{k+1} F_{k}
$$

where $F_{k}$ represents the $k$ th term of the sum in Eq. (17) (Pearson, 2009). 


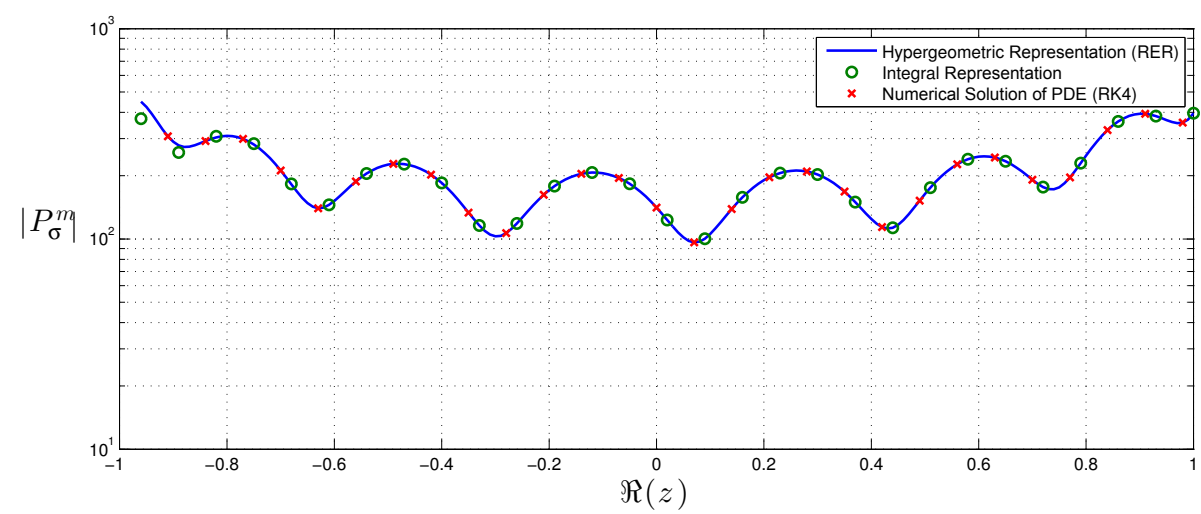

Figure 3. Comparison between three computation methods for $P_{\sigma}^{m}(z)$ with $m=3, \sigma=8.3843$ and $\Im(z)=0.0602$.
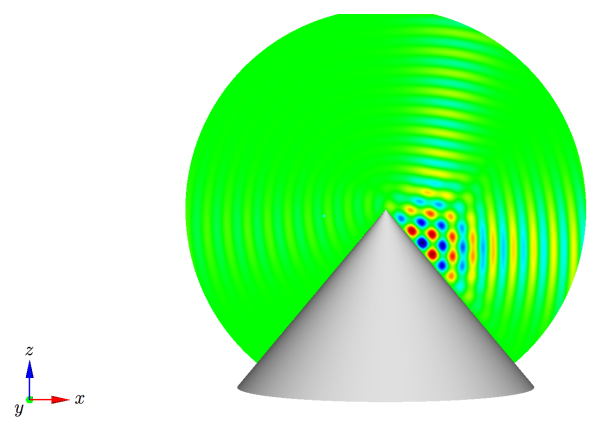

Figure 4. Diffraction of a complex-source beam by an acoustically soft circular cone with an outer half opening angle $\vartheta_{0}=140^{\circ}$ : Real part of the total near field in the region $r<10 \lambda$ in the $x z$ plane. The complex-valued source coordinate is given by $\boldsymbol{r}_{\mathrm{c}}=(3,0,-2) \lambda-$ $j(-7,0,0) \lambda$.

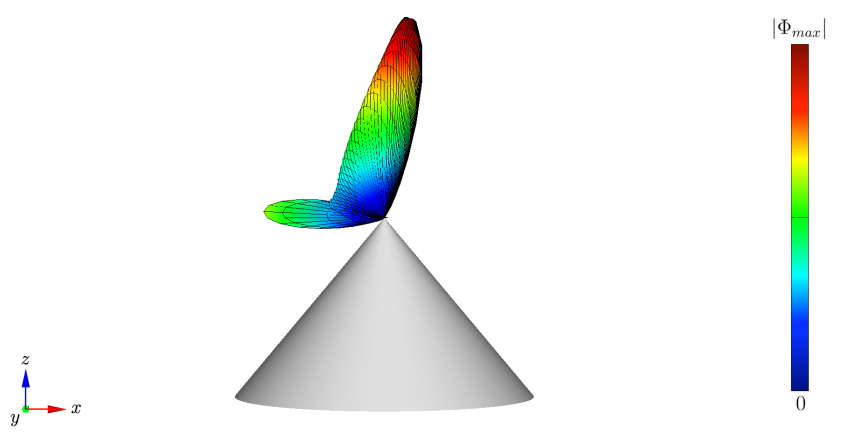

Figure 5. Radiation pattern of the total far field; for the other data see Fig. 4.

\section{Results}

\subsection{Numerical results for the associated Legendre functions for complex-valued arguments}

Figure 3 shows a comparison between the results of the three used methods by computing $\left|P_{\sigma}^{m}(z)\right|$ as a function of the real part at a constant imaginary part of $z$. While the hypergeo- metric representation by Ferrer and the purely numerical solution (RK4) deliver nearly similar results, the integral representation was found to become inaccurate nearby the boundaries. However, for evaluating $\left|P_{\sigma}^{m}(z)\right|$ at higher values of $\sigma$ and $m$ the RK4-method becomes unstable to an extent that even by choosing a denser meshing number the error could not be controlled. For instance, evaluating the associated Legendre function for $\vartheta=1.462-j 0.243, \sigma=51.847$ and $m=36$, the RK4 method needed $3.6627 \mathrm{~s}$ at a mesh number of 5000 and a rel. error of 4.3266, and $36.3594 \mathrm{~s}$ at a mesh number of 500000 and a rel. error of $4.3321 \times 10^{-4}$. For the same parameters, the evaluation of the hypergeometric representation was done in $0.0069 \mathrm{~s}$ on the same computer at a rel. error of $1.2486 \times 10^{-6}$. As a reference solution we used the MATLAB routine hypergeom which took $0.7610 \mathrm{~s}$.

In conclusion, for our set of parameters including eigenvalues of both $\sigma$ and $m$ up to 50, the hypergeometric representation by Ferrer stands out as the most suitable approach.

\subsection{Numerical evaluation of the near- and far fields}

Figure 4 represents the diffracted (total) field for an incident CSB with a waist at $\boldsymbol{r}_{\mathrm{w}}=(3,0,-2) \lambda$ (right side to the tip, travelling in the $-\hat{\boldsymbol{x}}$ direction). We clearly observe the reflected field and the shadowing effect of the cone. The radiation pattern of the total radiated far field, i. e., the scattered field plus the outwardly traveling part of the incident (CSB) field, for the same case is shown in Fig. 5.

Finally, in Fig. 6 (same configuration as before but different viewing angle) we see that beside the main lobe of the incident field there appear side lobes which are due to an interference between the incident and scattered fields.

\section{Conclusions}

The scattering of an arbitrarily oriented CSB by an acoustically soft or hard semi-infinite circular cone has been analysed using a spherical-multipole expansion. Among three different algorithms to compute the associated Legendre 


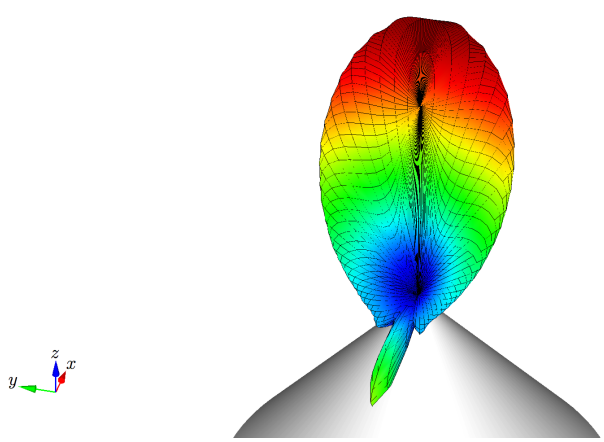

Figure 6. Radiation pattern of the total far field; same configuration but different viewing angle as in Fig. 5.

functions of the first kind for complex arguments that one based on Ferrer's hypergeometric representation was found to be most suitable. Convergent results of the total near and far fields have been obtained for arbitrary circular cones and directions of incidence. Future work will extend the application of this approach to other geometries, e.g. elliptic cones.

Acknowledgements. This work was supported by the Deutsche Forschungsgemeinschaft under Grant KL815/10-1\&2.

Edited by: R. Schuhmann

Reviewed by: two anonymous referees

\section{References}

Bowman, J. J., Senior, T. B. A., and Uslenghi, P. L. E.: Electromagnetic and acoustic scattering by simple shapes (revised printing), New York, Hemisphere Pub. Corp, 1987.

Brüns, H. and Klinkenbusch, L.: Electromagnetic diffraction and scattering of a complex-source beam by a semi-infinite circular cone, Adv. Radio Sci., 11, 31-36, doi:10.5194/ars-11-31-2013, 2013.

Katsav, M., Heyman, E., and Klinkenbusch, L.: Complex-source beam diffraction by an acoustically soft or hard circular cone, Proc. 2012 International Conference on Electromagnetics in Advanced Applications (ICEAA12), Cape Town, South Africa, 2-7 September 2012, 135-138, 2012.

Kijowski, M. and Klinkenbusch, L.: Eigenmode analysis of the electromagnetic field scattered by an elliptic cone, Adv. Radio Sci., 9, 31-37, doi:10.5194/ars-9-31-2011, 2011.

Klinkenbusch, L.: Electromagnetic scattering by semi-infinite circular and elliptic cones, Radio Sci., 42, RS6S10, doi:10.1029/2007RS003649, 2007.

Felsen, L. N.: Complex-source-point solutions of the field equations and their relation to the propagation and scattering of Gaussian beams, in: Symposia Matematica, Istituto Nazionale di Alta Matematica, Vol. XVIII, 40-56, London, Academic Press, 1976.

Orlob, S. and Peschel, U.: Complex source beam: A tool to describe highly focused vector beams analytically, Phys. Rev. A, 82, 063820, doi:10.1103/PhysRevA.82.063820, 2010.

Pearson, J.: Computation of hypergeometric functions, PhD Dissertation, University of Oxford, 2009.

Whittaker, E. T. and Watson, G. N.: A course of modern analysis, Fourth Edition Reprinted, Cambridge University Press, 2004. 